\title{
Techo de cristal en la Universidad. Si no lo veo no lo creo
}

\author{
Glass ceiling in the University. \\ If I don't see it don't believe it \\ Mauricio MATUS-LÓPEZ y Nazareth GALLEGO-MORÓN \\ Universidad Pablo de Olavide. Sevilla
}

Recibido: Febrero 2014

Aceptado: Abril 2014

\section{Resumen}

La carrera académica en la educación superior no es el mejor ejemplo de igualdad. Pese a que en otros sectores laborales los denominados techos de cristal han ido disminuyendo con los años, en la universidad prácticamente no se han producido modificaciones. Las estadísticas y la literatura confirman este fenómeno. Pese a ello, la percepción del problema es baja en el profesorado. El presente trabajo indaga sobre esta situación y determina si existen diferentes percepciones según perfiles y si estas diferencias son realmente significativas.

Aunque existe escasa literatura en revistas de alto impacto sobre la realidad española, los estudios nacionales tienen una amplia y reputada trayectoria. Por ello, en la primera parte de este trabajo se pone en valor una extensa revisión de los estudios nacionales y su inserción en el contexto internacional. Posteriormente se presentan los resultados del estudio aplicado. Se utiliza una metodología cuantitativa basada en una encuesta a 35 profesores/as de la Universidad Pablo De Olavide en la que se les pide el grado de acuerdo con una serie de afirmaciones sobre el techo de cristal. Los resultados son sometidos a test de contrastes no paramétricos para muestras pequeñas en función de las variables de sexo, edad, antigüedad, categoría y área.

Los resultados confirman algunas conclusiones de trabajos cualitativos a la vez que descartan otras. En primer lugar, se confirma una baja percepción del problema. El profesorado considera que el techo de cristal ocurre con mayor intensidad fuera del ámbito universitario. Asimismo, las diferencias entre las percepciones de mujeres y hombres son estadísticamente significativas, pero no así la relación entre el sexo, la antigüedad y la edad del profesorado. En tercer lugar, el profesorado más joven o con menor antigüedad percibe con menor intensidad este problema, lo que puede generar dificultades en la aceptación de políticas futuras de igualdad.

Palabas clave: discriminación de género, universidades, educación superior, actitudes, igualdad de oportunidades.

\footnotetext{
Abstract

Academic life is not a good example of equality. While in other sectors of employment the socalled glass ceilings have been declining over the years, at the University level nothing has changed. There are many impediments and/or barriers to career advancement for women in 
higher education. Literature and statistics confirm this phenomenon. Despite this, the perception of the problem is low among academic staff. The present work inquires about this situation and determines if there are different perceptions according to profiles and if these differences are really significant.

There is little literature about the Spanish situation in high-impact journals. National studies have a wide and reputable career. For this reason, this article made a wide review of the national studies related to glass ceiling phenomena and its insertion in the international context. The quantitative methodology used was based on a survey of 35 professors of the Pablo De Olavide University. They were asked to grade their opinion on a few sentences about the glass ceiling. The results were analysed with the nonparametric contrast test for small samples depending on the following variables: sex, age, seniority, category and area.

The results confirm previous conclusions of qualitative studies stated before while rejecting others. First, an underestimation perception of this problem was confirmed. Academic staff believes that glass ceiling occurs more in labour market outside the university environment. Second, differences between women and men are statistically significant but there is no relation between sex and age or seniority. Third, younger or less experienced teachers don't perceive this problem in its full importance. Therefore they might have difficulties accepting genderequality policies.

Keywords: gender discrimination, universities, higher education, attitudes, equal opportunities.

Las estadísticas de mercado de trabajo son concluyentes. Las mujeres escasamente acceden a las categorías profesionales más altas. Según datos de la EPA, casi la mitad $(45,6 \%)$ de la fuerza de trabajo ocupada está compuesta por mujeres, pero en la categoría de directoras y gerentes en empresas privadas representan menos de una tercera parte (30,4\%). Un estudio internacional de Grant Thornton (2013) muestra que la proporción de empresas en España que no cuentan con ninguna mujer en sus equipos directivos alcanza el 37\%. Superior a la media global del 31\%. Asimismo, sólo tres de las empresas del IBEX 35 cuentan con mujeres ejecutivas dentro de sus consejos de administración (InforPress - IESE 2013).

A este fenómeno se le conoce como techo de cristal (TC). Su descripción literal hace referencia a una barrera que se interpone en la parta alta de la carrera laboral de las mujeres y que tiene las características de ser invisible, acotada y sólida (Morrison et al. 1987). Invisible por cuanto no es percibida como tal por parte importante de la sociedad y porque no existen impedimentos explícitos discriminatorios contra las mujeres, sino que es mayoritariamente producto de determinados comportamientos sociales. Es acotada puesto que es relativamente fácil determinar en qué punto de la escalera profesional se produce este quiebre. Una especie de umbral sólido que impide romperlo. Tras dicho umbral no habría mujeres y por debajo de ese umbral, el problema sería menor o no existiría.

Sin embargo, el fenómeno no es tan simple. Aunque por una cuestión práctica se mantiene la denominación original, su interpretación se ha extendido más allá de la literalidad. Por ejemplo, tras esa barrera efectivamente hay presencia de mujeres aunque al coste de mostrar mejores resultados productivos que sus pares varones (Lyness y Heilman, 2006) y debiendo adoptar comportamientos diferentes al resto de 
mujeres y adaptarse a un medio masculinizado (Adams y Funk, 2012). Por otro lado, no parece ser una barrera localizable y fácil de identificar sino más bien un laberinto de cristal compuesto de una serie de caminos que no conducen a la cima organizacional (Berenguer et al., 1999). Además, y en esta misma línea, el TC se comporta como un tipo de mochila que va acumulando los obstáculos a lo largo de la vida laboral. Se une así este fenómeno al de los suelos pegajosos, vale decir, la sobre representación de las mujeres en la parte baja de la pirámide organizacional (Baxter y Wright, 2000).

Gran parte de los estudios que han abordado esta problemática ponen énfasis en las dimensiones socioculturales y educativas de la dimensión social del problema. Para ello, se enmarcan en un campo teórico paradigmático que desarrolla las manifestaciones machistas, androcéntricas y patriarcales que caracterizan la organización social. Reconociendo la importancia de dicho marco teórico, el presente trabajo no se desarrolla bajo este enfoque, sino que se limita a la interpretación de la evidencia cuantitativa.

\section{Techos de cristal en la Universidad}

La bibliografía en este campo es extensa. Una búsqueda rápida de artículos sobre esta temática en bases de datos bibliográficas internacionales entrega casi doscientos artículos solamente desde 2010 en adelante y además ubica a España como el tercer país productor de trabajos académicos sobre esta temática, sólo por detrás de Estados Unidos y Gran Bretaña ${ }^{1}$. No obstante, cuando la búsqueda se reduce a la educación universitaria, los resultados caen a una veintena ${ }^{2}$. Su totalidad en los últimos diez años y la mitad de ellos en los últimos seis. Esta cuestión es al menos interesante desde dos puntos de vista. Por una parte muestra el incremento reciente de estos trabajos, pero por otra, llama la atención que aún sea escaza la investigación en este tema.

En el ámbito internacional destaca el trabajo de Jackson y O’Callaghan (2009), quienes realizan una revisión de 66 investigaciones sobre techos de cristal en Estados Unidos, de las cuales 23 se centran en el sector universitario. Sus resultados identifican una serie de trabajos que explican las diferencias de salarios por la desigual distribución en categorías profesionales y destaca que aún tomando todos los factores de productividad que puedan afectar a hombres y mujeres, se sigue manteniendo un margen de promoción desfavorable para estas últimas (Ginther y Hayes, 1999). Es tal la solidez de estas barreras que para Bonawitz y Andel (2009), el techo se cristal en las

\footnotetext{
${ }^{1}$ Búsqueda por los conceptos completos “techo de cristal” o "glass cieling” en los apartados título o materia en las bases de datos de Scopus y de ProQuest, categorizados por año de publicación. Acceso a través de la plataforma de biblioteca de la Universidad Pablo de Olavide http: athenea.upo.es el 10/11/2013.

${ }^{2}$ Misma búsqueda que la anterior a través de la misma plataforma. Se restringió la búsqueda agregando como condición que incluya "higher education" o "university" en los apartados título o materia. Mismas bases de datos el 10/11/2013,
} 
universidades se transforma en un techo de cemento, sobre el que las mujeres apenas han podido rasguñar sobre la superficie.

Aunque el estado del arte que realizan estos autores para el caso de Estados Unidos es extensible a muchos países. Existe variedad en el avance de estos estudios. Pese a que la mayoría se encuentra aún en fase de diagnóstico de esta situación, hay otros como el caso de Holanda, en el que ya se evalúa la eficacia de políticas de intervención. En este sentido, Timmers, Willemsen y Tijdens (2010) constatan que de una quincena de políticas implementadas en las universidades holandesas, aquellas que involucraron la perspectiva cultural parecen haber sido las más eficaces en la reducción del TC. Como por ejemplo reuniones de análisis del problema, participación de mujeres en mesas de contratación, formación en género para los staff y realización de estudios de impacto de género de las decisiones de contratación y promoción.

Una revisión de las estadísticas de participación de mujeres y hombres en la academia en diez países, incluyendo España, muestra que se repite un mismo fenómeno: un descenso de la tasa de participación femenina a medida que se incrementa el nivel académico y lo contrario con la masculina (Guil, 2008).

El diagnóstico que Andreu (2002) realizó para España hace más de una década, sigue vigente. En los estudios de grado de las universidades españolas las mujeres son mayoría (54,0\%), al igual que en los Master Oficiales (54,6\% de la matrícula y 59,6\% de los egresos). Aún en las tesis doctorales leídas representan casi la mitad del total (48,6\%) (MECyD, 2012). No obstante, en el Personal Docente e Investigador (PDI) de las universidades públicas españolas, la representación de las mujeres cae al 38,6\% y además, se reduce aceleradamente a medida que el grado académico es mayor. En la parte baja de la escala actual representa en torno al $40 \%$, pero en el total de catedráticos/as son apenas el 20,5\%. Vale decir, existe un catedrático por cada 7 varones del PDI y una catedrática por cada 16 mujeres (MECyD, 2013).

$\mathrm{Al}$ contrario de lo que se suele pensar, la situación no ha variado mucho en los últimos años. En el periodo que va desde la Reforma Universitaria de 1983 a 1994, el porcentaje de mujeres catedráticas apenas aumentó en 1,8 puntos porcentuales (del $11 \%$ al 11,8\%). La década posterior, de 1994 a 2004 el incremento fue notablemente superior, impulsado por la creación de universidades, alcanzando hasta el $18 \%$. Sin embargo desde entonces hasta 2012-2013 (casi una década después) el incremento volvió a ser sólo de 2,5 puntos, hasta el 20,3\%. Aunque esta evolución pueda considerarse un avance, su proporcionalidad con respecto a la incorporación de las mujeres a la academia es prácticamente nula. En 1984 el porcentaje de hombres catedráticos sobre el total de varones del PDI era del 16\%, mientras que en las catedráticas era del 5,9\%. Treinta años después estos porcentajes apenas han variado. Los hombres muestran un leve descenso hasta el 14\% mientras que en las mujeres esta proporción incluso ha disminuido hasta el 5,6\% (INE, 1984; MECyD, 2014).

El futuro inmediato tampoco muestra signos de contrarrestar esta tendencia. Con el nuevo sistema de acreditación se eliminan muchas de las subjetividades locales, pero se mantienen las tasas que consolidan esta distribución. De acuerdo a los resultados oficiales de la Agencia Nacional de Evaluación de la Calidad y Acreditación (ANECA, 
2013), desde la implementación del sistema y hasta octubre de 2012, este organismo ha recibido un total de 24.145 solicitudes, de las que 8.041 corresponde a la figura de Catedrático/a de Universidad (CU). Las tasas de respuestas positivas casi no difieren entre mujeres y hombres (en torno al 70\% cada una), sin embargo, la proporción de solicitudes sí lo hace. Posiblemente la cuestión radica en las perspectivas o más bien dicho expectativas que puedan tener mujeres y hombres a la hora de solicitar la cátedra. De acuerdo a Alonso (2002), no existen diferencias significativas en las carreras profesionales de mujeres y hombres, no obstante la solicitud de cátedras es significativamente más baja en mujeres. Por ejemplo, si se considera que el mayor potencial de solicitudes provienen del PDI con categoría de Profesor Titular de Universidad, se puede estimar que las solicitudes de varones equivalen al 26,7\% de los varones titulares, mientras que entre las mujeres este porcentaje sólo llega al 21,5\% (ANECA, 2013). Así, si se continúa con esta dinámica en el tiempo difícilmente las proporciones finales cambien.

Aunque existe escasa bibliografía en revistas de alto impacto sobre esta temática en España, los estudios nacionales tienen una amplia y reputada trayectoria.

En el año 1997 el Centro de Investigaciones Sociológicas publicó un estudio diagnóstico basado en 254 encuestas a catedráticas en las universidades españolas (García y García, 1997). Sus resultados desvelaron la realidad y describieron sus percepciones. Entonces, pese a que las catedráticas sólo representaban el 9,59\% de las plazas de su categoría, no percibían tal desigualdad y consideraban que tanto hombres como mujeres gozaban de las mismas oportunidades y dificultades. Estas mismas autoras dirigieron un nuevo estudio seis años más tarde (García y García coord., 2001), cuando las catedráticas apenas habían aumentado hasta el $11 \%$. En este último trabajo se realizó la revisión de una quincena de trayectorias de mujeres en dicha categoría y otras tantas de hombres, en la que constaba que la situación prácticamente no había sufrido modificaciones y que las percepciones seguían siendo miopes (Alonso, 2002).

A estos trabajos le han seguido muchos otros, la mayoría basados en estudios de casos. Guil (2005, 2007) estudió la realidad en la Universidad de Sevilla; Ion, Durán y Bernabeu (2013) lo hicieron para ésta misma institución y la Universidad Autónoma de Barcelona; Morales, Luna y Esteban (2010) para la Universidad de Málaga y Tomás y Guillamon (2009) para cuatro universidades catalanas.

A todos estos cabe agregar algunos estudios más genéricos sobre las mujeres en las universidades, que aportan más información sobres este problema como son el trabajo de Guil, Solano y Álvarez (2005) para las universidades andaluzas, Alcalá, Guil y Pérez coord. (2005) y Torres y Pau (2011) para ciencia y tecnología o Callejo, Gómez y Casado (2004) y Grañeras y Gil coord. (2011) para el sistema educativo en su conjunto.

Un denominador común atraviesa los estudios revisados; la realidad va por un lado y la teoría por otro. Es decir, existe una brecha entre lo "oficialmente escrito" y lo "realmente vivido" (Ion et al., 2013). En la norma, la igualdad es indiscutible, pero en la práctica es "natural” (Callejo et al., 2004). De esta manera, no es de extrañar los 
malos resultados en la evaluación de los planes de igualdad en las universidades (De los Cobos, 2012).

Casi todos los estudios muestran que la esta situación no ha sido valorada como un problema en toda su dimensión y por lo tanto no se encuentra entre las principales preocupaciones del sistema educativo (Andreu, 2002; Pastor et al., 2010; Callejo et al., 2004). Esto es esencial, ya que como señala Alonso (2002): <<las posibilidades de que esta situación de flagrante desigualdad evolucione dependerá en buena medida de que se comience siquiera a percibirla y reconocerla $>>$ (p.73). De ello dependen el éxito de las políticas o medidas de igualdad destinadas a eliminar los techos de cristal en las universidades (Jackson et al., 2009; Timmers et al., 2010).

En esta cuestión radica la relevancia de este artículo. La investigación académica aplicada entrega suficientes argumentos que corroboran la existencia de los techos de cristal en la universidad y apuntan a la escasa toma de conciencia del profesorado como una traba para superar esta situación. Sin embargo, para implementar políticas de concienciación o de intervención es necesario tener un mapa claro de cuáles son las percepciones del profesorado y particularmente si realmente existen estas diferencias en las percepciones por sexo, categoría o área de conocimiento. Sin esta información la efectividad de políticas de concienciación o intervención será muy baja y por supuesto ineficiente. En el presente trabajo se pretende avanzar en este punto.

\section{Planteamiento del problema}

La literatura nacional como internacional refleja que las mujeres están subrepresentadas en los estratos más altos de la jerarquía académica.

Lo cierto es que la igualdad de oportunidades es esencial desde el punto de vista moral, socioeducativo y económico ya que la discriminación no sólo desestabiliza la paz social sino que impacta negativamente en la eficiencia del principal factor productivo; el capital humano. La universidad se sitúa en este sentido en la propia especificidad que surge de representar la cumbre de la pirámide educacional y el paradigma del avance en el conocimiento.

Las investigaciones y la recolección de información vía cuestionarios y/o entrevistas en profundidad, llevan a la conclusión de que el problema no es dimensionado en su totalidad por el personal académico. Se sugiere que hombres y mujeres perciben de manera distinta esta realidad y se señala una amplia gama de causas, que van desde obstáculos personales y sociales a formales y organizacionales.

Por ello cabe preguntarse ¿Existe realmente tal subestimación del problema? ¿Son los hombres menos sensibles a esta realidad que las mujeres? ¿ $\mathrm{i}$ los catedráticos con respecto a los titulares? ¿Son las jóvenes más conscientes del problema? ¿Es un mismo grupo social el que no lo percibe? ¿Son estas diferencias, si las hay, realmente significativas?

Uno de los principales problemas a la hora de responder estas preguntas es que la recolección de datos cuantitativos en esta particular materia suele ser frustrante en 
términos de respuestas recabadas, lo que impide muchas veces contrastar empíricamente las diferencias.

En el presente trabajo, gracias a la utilización de pruebas no paramétricas se solventa esta dificultad y se contrastan diferencias de perfil relevantes. Los resultados, como se verá en el apartado de análisis, rechazan creencias arraigadas a la vez que confirman algunas presunciones más recientes.

\section{Método}

\section{Hipótesis principal}

La hipótesis principal que se plantea consiste en que ni el sexo del profesorado ni su categoría profesional influyen en la percepción sobre el problema de techo de cristal que se constata en los datos oficiales.

\section{Muestra}

Los resultados que se presentan en los siguientes apartados corresponden a un estudio realizado en la Universidad Pablo de Olavide (UPO) durante el año 2013, cuyo objetivo fue constatar estadísticamente si existían diferencias en las percepciones sobre el techo de cristal entre el profesorado funcionario de la Universidad según distintas características de sus perfiles.

El universo de la encuesta es el profesorado funcionario de la universidad, cuyo listado fue facilitado por la Oficina de Recursos Humanos de la UPO de acuerdo a la información de mayo de este año. Esta Universidad, fundada en 1997 tiene como característica ser la segunda más joven de España, con un promedio de edad del profesorado de sólo 42 años, frente a los 47 años de media nacional y una tasa de feminización del PDI algo superior al promedio; 41\% frente a 38,6\% (MECyD, 2013).

En un ranking de igualdad la UPO ocupa el lugar vigésimo de un total de 70 universidades (Elizondo, Novo y Silvestre, 2007). De acuerdo a los datos facilitados por Recursos Humanos, la UPO cuenta de 257 profesores/as funcionarios, el 33,1\% mujeres, de los que 66 son catedráticos/as de universidad, un 16,6\% mujeres. Los funcionarios/as de escuela alcanzan el 2,7\%.

La muestra no es aleatoria. Se distribuyó la encuesta al total del profesorado funcionario de la universidad y se obtuvo una tasa de respuesta del $14,5 \%$, compuesta en un $40 \%$ por mujeres y un $60 \%$ por hombres. La baja respuesta del profesorado coincide con otros estudios como el de Guil et al (2005) para el total andaluz. Ello tiene una fortaleza y una limitación. La fortaleza es que puede ser interpretado como un indicador de la sensibilidad (positiva o negativa) con esta temática de parte de distintos grupos del profesorado y la debilidad es que los resultados que se obtienen deben ser considerados con las restricciones de extrapolación que la muestra tiene. 


\section{Instrumento}

La herramienta utilizada fue una encuesta simple constituida por ocho aseveraciones sobre desigualdad y techo de cristal en la Universidad. Estas afirmaciones fueron formuladas de acuerdo a los resultados de la revisión de la literatura. La persona encuestada seleccionó el grado de acuerdo con cada una, en una escala de uno a cinco. Las primeras tres preguntas contienen información sobre la percepción del techo de cristal, las siguientes cuatro sobre obstáculos para acceder a la categoría más alta (personales/culturales y de la organización) y la última, sobre impacto de las leyes de igualdad aprobadas en los últimos años. Estas son:

- P1. En el mercado de trabajo español existen más barreras para las mujeres que para los hombres a la hora de alcanzar las categorías profesionales más altas.

- P2. En la Universidad existen más barreras para las mujeres que para los hombres a la hora de alcanzar las categorías profesionales más altas (Cátedra).

- P3. En los últimos 10 años han disminuido las barreras de acceso para las mujeres a las categorías profesionales más altas en la Universidad (Cátedra).

- P4. El matrimonio es un factor que dificulta el acceso a las categorías profesionales más altas en la Universidad (Cátedra).

- P5. Tener hijos/as es un factor que dificulta el acceso a las categorías profesionales más altas en la Universidad (Cátedra).

- P6. Tener personas dependientes es un factor que dificulta el acceso a las categorías profesionales más altas en la Universidad (Cátedra).

- P7. La cooperación y apoyo entre hombres es una barrera para que las mujeres accedan a las categorías más altas en la Universidad (Cátedra).

- P8. Las leyes de igualdad aprobadas en España y Andalucía han favorecido el acceso a las categorías profesionales más altas en la Universidad (Cátedra).

\section{Procedimiento}

El análisis de las respuestas está condicionado por la utilización de escalas de Lickert en las preguntas y por el tamaño de la muestra. Como señala Berlanga y Rubio (2012), tras una exhaustiva revisión de la literatura metodológica, para muestras pequeñas como esta se debe recurrir a pruebas no paramétricas. En concreto, se ha utilizado la Prueba U de Mann-Whitney para la comparación de las variables Sexo (hombre/mujer) y Categoría (titular/catedrático) y la prueba $\mathrm{H}$ de Kruskal-Wallis para las variables con más de dos categorías: Edad (0 a 40 años, 50 a 60 años, más de 60 años), Área (Empresa, Experimental, Sociales y Humanidades) y Antigüedad como funcionario (1-5 años, 6 a 15 años, 16 y más años). Previamente, se contrasta la correlación entre las variables a través del test de Spearman.

\section{Limitaciones}

La principal limitación de este estudio tiene que ver con el tamaño de la muestra y las tasas de respuesta. Al no poder implementar una muestra aleatoria, se puede suponer que las personas que han respondido a la encuesta estén particularmente 
interesadas o sensibilizadas en esta problemática. Pero no es posible determinar si las personas que respondieron la encuesta (en comparación con quienes no lo hicieron) sienten la desigualdad como una realidad o por el contrario pretenden negarla. En cualquier caso, aunque limitada, la evidencia presentada es un aporte que se complementa al marco teórico y aplicado del estudio de la desigualdad en otras universidades españolas.

\section{Resultados}

Las tasas de respuesta fueron heterogéneas según la variable considerada. Las mujeres respondieron más a la encuesta que los hombres: $16,5 \%$ frente a $12,2 \%$ y los catedráticos más que los titulares: 22,7\% y 11,4\% respectivamente. Asimismo, por área de conocimiento, Ciencias Experimentales y Ciencias Empresariales registraron las tasas más elevadas y las más bajas, Deportes, Ciencias Sociales y Humanidades. De acuerdo a la literatura, se construyó una variable denominada Área, que dejó en una categoría separada a Cs. Experimentales, en otra a Cs Empresariales y en una tercera, denominada Cs. Humanas, al resto.

Las características de la muestra son las que se presentan en la Tabla 1. La primera columna corresponde a las variables y sus categorías. En las siguientes, el valor de los resultados de acuerdo con cada una de las afirmaciones del cuestionario ( 0 totalmente en desacuerdo y 5 totalmente de acuerdo).

\begin{tabular}{lrrrrrrrr}
\hline & P1 & P2 & P3 & P4 & P5 & P6 & P7 & P8 \\
\hline Hombre & 3,76 & 2,24 & 3,57 & 2,90 & 3,52 & 3,81 & 2,35 & 3,30 \\
\hline Mujer & 4,07 & 3,21 & 3,14 & 2,79 & 3,93 & 4,07 & 2,85 & 2,54 \\
\hline$<$ 40 años edad & 2,80 & 1,40 & 4,00 & 2,20 & 3,00 & 3,20 & 1,80 & 3,20 \\
\hline 41 a 50 años edad & 4,12 & 2,71 & 3,76 & 2,71 & 3,88 & 4,12 & 2,73 & 3,07 \\
\hline$>$ 50 años edad & 4,00 & 3,00 & 2,69 & 3,31 & 3,69 & 3,92 & 2,62 & 2,85 \\
\hline Categoría Titular & 3,85 & 2,50 & 3,75 & 2,55 & 3,55 & 3,90 & 2,39 & 3,17 \\
\hline Categoría Catedrático/a & 3,93 & 2,80 & 2,93 & 3,27 & 3,87 & 3,93 & 2,73 & 2,80 \\
\hline Área Empresa & 4,00 & 2,67 & 3,22 & 2,89 & 3,11 & 3,56 & 2,33 & 3,00 \\
\hline Área Sociales & 3,90 & 2,70 & 3,20 & 3,10 & 3,80 & 3,90 & 2,90 & 2,50 \\
\hline Área Experimentales & 3,93 & 2,60 & 3,60 & 2,67 & 3,93 & 4,13 & 2,46 & 3,29 \\
\hline 0-5 años laborales & 4,00 & 2,20 & 4,00 & 2,00 & 2,20 & 3,00 & 1,80 & 3,50 \\
\hline 6 a 15 años laborales & 3,89 & 2,58 & 3,47 & 2,79 & 4,00 & 4,16 & 2,47 & 3,11 \\
\hline$>15$ años laborales & 3,82 & 2,91 & 3,00 & 3,36 & 3,82 & 3,91 & 3,00 & 2,64 \\
\hline Promedio & 3,89 & 2,63 & 3,40 & 2,86 & 3,69 & 3,91 & 2,55 & 3,00 \\
\hline Desviación & 0,99 & 1,31 & 1,22 & 1,35 & 1,18 & 0,95 & 1,12 & 1,00 \\
\hline
\end{tabular}

Tabla 1. Indicadores descriptivos de los resultados. UPO 2013.

Como se puede apreciar en la Tabla I, existen variables que determinan los valores medios más bajos y más altos. Algunos de ellos parecen apuntar a perfiles similares, como son la antigüedad y la edad de la persona. El test de correlación no paramétrico de Spearman permite confirmar ciertas relaciones significativas (Tabla 2). 


\begin{tabular}{|c|c|c|c|c|c|c|}
\hline & & Sexo & Edad & Categoría & Área & Antigüedad \\
\hline \multirow{3}{*}{ Sexo } & Coef. & 1 & $-0,184$ & $-0,236$ &,$- 382^{*}$ & $-0,212$ \\
\hline & Sig. & . & 0,291 & 0,173 & 0,026 & 0,221 \\
\hline & $\mathrm{N}$ & 35 & 35 & 35 & 34 & 35 \\
\hline \multirow{3}{*}{ Edad } & Coef. & $-0,184$ & 1 &, $564^{* *}$ & $-0,133$ & ,362* \\
\hline & Sig. & 0,291 & . & 0 & 0,454 & 0,033 \\
\hline & $\mathrm{N}$ & 35 & 35 & 35 & 34 & 35 \\
\hline \multirow{3}{*}{ Categoría } & Coef. & $-0,236$ &, $564^{* *}$ & 1 & $-0,277$ & ,764 \\
\hline & Sig. & 0,173 & 0 & . & 0,112 & 0 \\
\hline & $\mathrm{N}$ & 35 & 35 & 35 & 34 & 35 \\
\hline \multirow{3}{*}{ Área } & Coef. &,$- 382^{*}$ & $-0,133$ & $-0,277$ & 1 & 0,106 \\
\hline & Sig. & 0,026 & 0,454 & 0,112 & . & 0,552 \\
\hline & $\mathrm{N}$ & 34 & 34 & 34 & 34 & 34 \\
\hline \multirow{3}{*}{ Antigüedad } & Coef. & $-0,212$ & ,362 &, $764^{* *}$ & 0,106 & 1 \\
\hline & Sig. & 0,221 & 0,033 & 0 & 0,552 & . \\
\hline & $\mathrm{N}$ & 35 & 35 & 35 & 34 & 35 \\
\hline
\end{tabular}

Tabla 2. Tabla de correlaciones entre variables. UPO 2013.

Las correlaciones son moderadas entre sexo-área y edad-antigüedad, mayor entre edad-categoría y fuerte entre categoría-antigüedad. Todas ellas significativas estadísticamente. La explicación es que el ascenso a catedrático requiere de una inversión en producción científica y docente, para lo que la antigüedad es necesaria, condicionando la edad de los profesores que acceden a esta categoría. En relación al área, la agrupación fue construida considerando la proporción de mujeres, por lo que no aporta información relevante la correlación.

\begin{tabular}{llcccccccc}
\hline & & P1 & P2 & P3 & P4 & P5 & P6 & P7 & P8 \\
\hline \multirow{3}{*}{ Sexo } & Mann-Whitney & 115 & $88^{* *}$ & 126,5 & 139,5 & $102^{* *}$ & 127,5 & 103 & $69^{* *}$ \\
& Sig. & 0,25 & 0,041 & 0,465 & 0,795 & 0,079 & 0,435 & 0,302 & 0,018 \\
& Mann-Whitney & 141,5 & 136,5 & $92^{* *}$ & 105 & 142 & 149,5 & 119 & 109 \\
\hline \multirow{3}{*}{ Edad } & Sig. & 0,762 & 0,643 & 0,041 & 0,123 & 0,757 & 0,984 & 0,548 & 0,322 \\
& Kruskal-Wallis & $6,729^{*}$ & 5,816 & $8,097^{*}$ & 2,882 & 1,118 & 1,281 & 2,849 & 0,674 \\
& Sig. & 0,035 & 0,055 & 0,017 & 0,237 & 0,572 & 0,527 & 0,241 & 0,714 \\
\hline \multirow{3}{*}{ Categoría } & Kruskal-Wallis & 0,578 & 0,012 & 0,657 & 0,586 & 1,711 & 1,182 & 2 & 3,33 \\
& Sig. & 0,749 & 0,994 & 0,72 & 0,746 & 0,425 & 0,554 & 0,368 & 0,189 \\
& Kruskal-Wallis & 0,37 & 0,758 & 2,762 & 3,696 & $6,38^{* *}$ & 3,895 & 3,503 & 2,343 \\
\hline \multirow{2}{*}{ Área } & Sig. & 0,831 & 0,684 & 0,251 & 0,158 & 0,041 & 0,143 & 0,174 & 0,31 \\
& Mann-Whitney & 115 & $88^{* *}$ & 126,5 & 139,5 & $102^{* *}$ & 127,5 & 103 & $69^{* *}$ \\
& Sig. & 0,25 & 0,041 & 0,465 & 0,795 & 0,079 & 0,435 & 0,302 & 0,018 \\
\hline
\end{tabular}




\begin{tabular}{lllllccccc}
\hline \multirow{3}{*}{ Antigüedad } & Mann-Whitney & 141,5 & 136,5 & $92^{* *}$ & 105 & 142 & 149,5 & 119 & 109 \\
& Sig. & 0,762 & 0,643 & 0,041 & 0,123 & 0,757 & 0,984 & 0,548 & 0,322 \\
& Kruskal-Wallis & $6,729^{*}$ & 5,816 & $8,097^{*}$ & 2,882 & 1,118 & 1,281 & 2,849 & 0,674 \\
\hline
\end{tabular}

Tabla 3. Contrastes de diferencias. UPO 2013.

Teniendo esto en cuenta, se contrastaron estadísticamente las diferencias en las valoraciones de la Tabla I de acuerdo a las pruebas U de Mann-Whitney y $\mathrm{H}$ de Kruskal-Wallis (Tabla 3).

La mayoría de las diferencias no son estadísticamente significativas, con algunos perfiles destacables. Se realizaron varias pruebas con variables creadas en base a estas, cuyos resultados no entregan información relevante.

\section{Análisis}

En el marco de la evidencia bibliográfica, el presente trabajo confirma estadísticamente algunas de las conclusiones previas como la baja percepción de esta problemática dentro de la Universidad y la diferencia entre distintos perfiles del profesorado.

Los profesores y profesoras encuestadas se encuentran en su mayoría de acuerdo con las afirmaciones sobre desigualdad de género y techo de cristal, aunque los apoyos son moderados. Existe mayor acuerdo en la existencia del techo de cristal en el mercado de trabajo en general y que en la Universidad se ha avanzado poco en los últimos diez años. En cuanto a las barreras, concitan acuerdo la de tener personas dependientes a cargo y en menor medida, hijos/as. En relación a las leyes de igualdad, parece ser que éstas han ayudado poco a la resolución de este problema. Por el contrario, es bajo el reconocimiento de la existencia del techo de cristal en la misma Universidad, tampoco se considera el matrimonio como un problema importante en el acceso a la cátedra y por último, es escaso el apoyo que concita la existencia de procesos de cooptación como causa.

Las respuestas varían de forma importante según los perfiles estudiados. Con ello, se perciben dos variaciones importantes en las apreciaciones. Un reconocimiento más fuerte del problema entre los catedráticos y las mujeres mayores y uno menor entre las jóvenes o con baja antigüedad en el funcionariado. En un análisis de correlación se contrastó positivamente la correlación entre estas variables (edad, antigüedad y categoría), por lo que estos perfiles apuntan en la misma dirección.

En cuanto a las brechas entre los promedios de acuerdo con las afirmaciones, estas son más amplias cuando se considera el criterio de la edad o la antigüedad, ambas correlacionadas. Las personas mayores de 40 años o con una antigüedad en el funcionariado mayor de cinco años respaldan con mayores valores que las más jóvenes su nivel de acuerdo con casi todas las preguntas. Algo similar se produce con las dos primeras aseveraciones, relativas a la existencia del techo de cristal, que los profesores/as de empresa valoran menos que el resto. En cuanto a las diferencias por sexo, las mujeres muestran mayor grado de acuerdo con la existencia de techo de 
cristal y sus barreras que los hombres, también valoran menos positivamente su evolución en el tiempo y muestran poco acuerdo con el posible impacto positivo de las leyes de igualdad.

De todos estos resultados sólo siete resultan estadísticamente significativos. En primer lugar, las diferencias entre mujeres y hombres en tres de las siete afirmaciones fueron respaldadas. En concreto, la existencia del problema en la Universidad, la barrera que implica tener hijos/as y la ineficacia de la ley de igualdad para combatir el techo de cristal. Otra variable interesante en la contrastación empírica fue la edad, que señala que las jóvenes perciben menos problemas que las mayores en términos de existencia de los techos de cristal y una mejor valoración del cambio en los últimos diez años en la universidad. En sentido contrario, las peores percepciones de los/las catedráticos/as sobre las de los/as titulares acerca de la evolución reciente también es respaldada estadísticamente. Lo que concuerda con otra diferencia significativa en este grupo como es la mayor percepción del problema en el mercado de trabajo de parte de los/as catedráticos/as. Por último, destacar la significancia de las diferencias en la percepción más negativa de las personas con mayor antigüedad acerca de los hijos/as como barrera al techo de cristal.

Respondiendo explícitamente a las preguntas realizadas al comienzo de este trabajo, se puede decir que en promedio existe una subestimación del problema. Evidentemente no es posible establecer objetivamente qué grado de acuerdo con las aseveraciones coincidiría con la realidad descrita por las estadísticas oficiales, pero parece ser que los valores obtenidos en la encuesta son sensiblemente bajos para algunos grupos. Así, se concluye con respaldo estadístico, que las mujeres perciben con mayor intensidad la discriminación del techo de cristal en la academia, mientras que los hombres reconocen menos esta realidad. De igual manera, las personas con la categoría de catedrático/a se mostraron más de acuerdo con la existencia de las barreras a las mujeres, en comparación con los /as titulares. En este mismo sentido, las diferencias entre las personas mayores, con mayor percepción del problema y sus barreras, y las más jóvenes, con percepciones más optimistas, mostraron ser estadísticamente significativamente en varios aspectos.

En resumen, se puede concluir que existen evidencia estadística que respalda una menor percepción del problema por parte de los hombres (en línea con la evidencia que sugiere la bibliografía), de las personas más jóvenes, con menor antigüedad en el funcionariado y en la escala de titular, pero que estas contrastaciones no son extensibles a las diferencias por área de conocimiento.

\section{Conclusiones}

Una visión general de los resultados de este trabajo permite concluir cuatro cuestiones. En primer lugar, salvo el reconocimiento del techo de cristal como un problema del mercado de trabajo genérico y la responsabilidad de personas dependientes como barrera para acceder a la categoría más alta, son más bien bajos los niveles de acuerdo con las otras afirmaciones, destacando particularmente la baja 
aceptación de la existencia de este problema en la misma Universidad y/o la existencia de procesos de cooptación en ella.

En segundo lugar, la antigüedad y/o la edad de las personas hacen que difieran bastante las apreciaciones. Las brechas entre jóvenes y mayores juegan a favor de una subvaloración de la problemática por quienes han ingresado más tarde a la Universidad.

Tercero, las mujeres perciben de forma más negativa las aseveraciones sobre el techo de cristal, en comparación con los hombres. Esta diferencia tiene respaldo estadístico. Se podría pensar que esta relación no tiene que ver con el sexo sino con la menor edad o antigüedad promedio de las mujeres y corresponderse con el punto anterior. Sin embargo, aunque las mujeres de la muestra son en promedio más jóvenes y llevan menos tiempo como funcionarias que los hombres, esta diferencia en edad y antigüedad no llega a ser estadísticamente significativa.

La revisión bibliográfica y estadística realizada previamente señala la existencia de este problema en la Universidad y más aún, afirma que no ha mejorado en las últimas décadas y que no se espera que lo haga en el futuro cercano.

La experiencia internacional demuestra que para ello es necesario que la misma organización tome conciencia del problema y que implemente medidas encaminadas a reducirlo.

En el caso estudiado, la opinión del profesorado es bastante heterogénea, aunque es posible identificar ciertas diferencias con respaldo estadístico. Existe acuerdo con la existencia de techos de cristal en el mercado de trabajo, pero su reconocimiento dentro del espacio académico es bajo. Las mujeres tienen percepciones más negativas sobre este fenómeno que los hombres. Curiosamente, por edad o antigüedad, son las personas jóvenes las que menos perciben la existencia de esta problemática. Esto último resulta trascendente a la hora de evaluar la igualdad de oportunidades en el ámbito universitario. Si la realidad apunta a una desigualdad de género y las personas que participan en este proceso no lo perciben, es complejo y poco probable que se supere.

\section{Referencias bibliográficas}

ADAMS, R. y FUNK, P. (2012). Beyond the glass ceiling: Does gender matter? Management Science, 58 (2), 219-235.

ALCALÁ, P., GUIL, A. y PÉREZ, E. (2005) (coord.). Mujer y Ciencia. La situación de las mujeres en el sistema educativo de ciencia y tecnología en España. Madrid: FECYT.

ALONSO, M. J. (2002). Las académicas. Profesorado universitario y género. Revista de educación, 328, 465-475.

ANDREU, S. (2002). La carrera académica por género (a propósito de dos investigaciones recientes). Revista complutense de educación, 13(1), 13-31. 
ANECA (2013). Memoria de las actividades Enero-Octubre 2012. Madrid: Agencia Nacional de Evaluación de la Calidad y Acreditación. Recuperado de www.aneca.es/content/download/12700/157565/file/memoria_eneoct_2012.pdf el $10 / 12 / 2013$

BAXTER, J. y WRIGHT, E. O. (2000). The glass ceiling hypothesis: A comparative study of the United States, Sweden, and Australia. Gender And Society, 14(2), 275294. DOI: $10.2307 / 190275$

BERENGUER, G., CASTELLVÍ, J. M., CERVER, E., JUAN, C., TORCAL, R. y DE LA TORRE, A. (1999). El laberinto de cristal. Un estudio sobre el acceso de las mujeres a los puestos de dirección en las cooperativas de trabajo asociado valencianas. Dificultades y oportunidades. Valencia: FVECTA.

BERLANGA, V. y RUBIO, M. (2012). Clasificación de pruebas no paramétricas. Revista d'Innovació i Reserca en Educació, 5(2), 101-113.

BONAWITZ, M. y ANDEL, N. (2009). The Glass Ceiling Is Made of Concrete: The Barriers to Promotion and Tenure of Women in American Academia. Forum On Public Policy Online.

CALLEJO, J., GÓMEZ, C. y CASADO, E. (2004). El techo de cristal en el sistema educativo español. Madrid: UNED Ediciones.

DE LOS COBOS, F. (2012). Planes de igualdad en las universidades españolas. Análisis de contenido desde las ciencias sociales. Barataria: revista castellanomanchega de ciencias sociales, 14, 117-130.

ELIZONDO, A., NOVO, A. y SILVESTRE, A. (2007). La presencia de mujeres y hombres en las universidades españolas. Aequalitas: Revista jurídica de igualdad de oportunidades entre mujeres y hombres, 21, 25-34.

GARCÍA DE LEÓN, M. A. y GARCÍA DE CORTÁZAR, M. L. (1997). Mujeres en minoría: una investigación sociológica sobre las catedráticas de universidad en España. Madrid: Centro de Investigaciones Sociológicas (CIS).

GARCÍA DE LEÓN, M. A. y GARCÍA DE CORTÁZAR, M. L. (Coord.) (2001). Las académicas. Profesorado universitario y género. Madrid: Instituto de la Mujer,

GINTHER, D. K., y HAYES, K. J. (1999). "Gender differences in salary and promotion in the humanities", The American Economic Review, vol. 89(2), 397402.

GRAÑERAS, M. y GIL, N. (Coords.) (2011). Mujeres en cargos de representación del Sistema Educativo II. Mujeres en la educación, 14, Madrid: Instituto de la Mujer.

GRANT THORNTON (2013). Presencia de mujeres en puestos directivos: retroceso en España. Recuperado de http://www.grantthornton.es/publicaciones/estudios/Grant-Thornton-Estudio-IBR2013-mujer-en-puestos-directivos.pdf 10/01/2014. 
GUIL, A. (2005). Techos de Cristal en la Universidad Hispalense. Informe final del proyecto del Plan Nacional I+D+i 2001-2004 Sevilla, Universidad de Sevilla. Recuperado de: http://www.amit-es.org/assets/files/publi/ana_guil_2004.pdf 03/08/2013

GUIL, A. (2007). Docentes e investigadoras en las universidades españolas: visibilizando techos de cristal. Revista de investigación educativa, 25 (1), 111-132.

GUIL, A. (2008). Mujeres y ciencia, techos de cristal. Eccos Revista Científica, 10(1), 213-232.

GUIL, A., SOLANO, A. y ALVAREZ, M. (2005). La situación de las mujeres en las universidades públicas andaluzas. Sevilla: Consejo Económico y Social de Andalucía.

INE (1984). Estadísticas de la Enseñanza en España. Madrid.

INFORPRESS \& IESE (2013). El peso de las mujeres en los consejos del Ibex 35. Recuperado de http://www.culturarsc.com/Genero/Informe_Peso_mujeres_consejos_Ibex35.pdf el 03/01/2014.

ION, G., DURÁN, M. y BERNABEU, M. D. (2013). El profesorado y su percepción sobre la igualdad de género en la universidad. Revista Complutense de Educación, 24(1), 123-140. DOI: 10.5209/rev_RCED.2013.v24.n1.41194

JACKSON, J. L. y O'CALLAGHAN, E. M. (2009). What do we know about glass ceiling effects? A taxonomy and critical review to inform higher education research. Research In Higher Education, 50(5), 460-482. DOI: 10.1007/s11162009-9128-9

LYNESS, K. y HEILMAN, M. (2006). When fit is fundamental: Performance evaluations and promotions of upper-level female and male managers. Journal of Applied Psychology, 91(4), 777-785. DOI: 10.1037/0021-9010.91.4.777

MECyD (2012). Datos y cifras del sistema universitario español. Curso 2012-2013. Madrid. Ministerio de Educación, Cultura y Deporte.

MECyD (2013). Estadística de personal al servicio de las universidades. PDI. Curso 2011-2012. Madrid: Ministerio de Educación, Cultura y Deporte.

MECyD (2014). Datos Básicos del Sistema Universitario Español. Curso 2013/2014. Recuperado de: http://www.mecd.gob.es/educacion-mecd/areaseducacion/universidades/estadisticas-informes/datos-cifras.html el 06/05/2014.

MORALES, M.J, LUNA, M.J. y ESTEBAN, A.I. (2010). Diagnóstico de paridad en la Universidad: análisis a través de indicadores. Revista de Universidad y Sociedad del Conocimiento, 7(2), 1-14.

MORRISON, A. M., WHITE, R.P. y VAN VELSOR, E. (1987). Breaking the Glass Ceiling: Can Women Reach the Top of America's Largest Corporations? New York: Addison-Wesley. 
PASTOR, I., BELZUNEGUI, A., MORENO, B. y MAÑAS, C. (2010). La igualtat d'oportunitats a la universitat: les percepcions del PDI. Papers: revista de sociología, 95(2), 457-481.

TIMMERS, T., WILLEMSEN, T. y TIJDENS, K. (2010). Gender diversity policies in universities: a multi-perspective framework of policy measures. Higher Education, 59(6), 719-735. DOI: 10.1007/s10734-009-9276-z.

TOMÁS, M. y GUILLAMON, C. (2009). Las barreras y los obstáculos en el acceso de las profesoras universitarias a los cargos de gestión académica. Revista de educación, 350, 253-275.

TORRES, O. y PAU, B. (2011). "Techo de cristal" y "suelo pegajoso": La situación de la mujer en los sistemas alemán y español de ciencia y tecnología. CTS: Revista iberoamericana de ciencia, tecnología y sociedad, 6(18), 35-59.

Agradecimientos:

Se agradece a la Tesinanda del Grado de Administración de Empresas, Celia Casado Godoy por su cooperación en la recolección de datos utilizados en el presente artículo.

\section{Correspondencia con los autores}

Mauricio MATUS-LÓPEZ

Dpto. Economía, Métodos Cuantitativos e Historia Económica

Universidad Pablo de Olavide

Sevilla, España

Tel. (34) 954977331

e-mail: mmatlop@upo.es

Nazareth GALLEGO-MORÓN

Doctorado en Desarrollo y Ciudadanía

Universidad Pablo de Olavide

Sevilla, España

Tel. (34) 665964995

e-mail: nazareth.gallego@gmail.com 